\title{
Improved RecA-assisted fluorescence assay for DNA strand exchange reaction
}

\author{
Oleg Kaboev ${ }^{1}$, Ludmila Luchkina ${ }^{1}$, Valery Shalguev ${ }^{1}$, Yury Andreichuk ${ }^{2}$, \\ Vladimir Kulikov², Alexander Kozarenko ${ }^{2}$, and Vladislav Lanzov ${ }^{1}$ \\ ${ }^{1}$ Petersburg Nuclear Physics Institute of RAS, Gatchina and ${ }^{2}$ HELIX Research \\ Company, St. Petersburg, Russia
}

BioTechniques 40:736-738 (June 2006)

doi $10.2144 / 000112195$

Bacterial RecA protein and its archaeal and eukaryotic homologs, RadA and Rad51, respectively, are ATP-dependent filamenting DNA transferases which play a key role in homologous recombination (HR) and many other processes of DNA metabolism (1-3). In the presence of ATP, RecA forms a right-handed helical filament on single-stranded DNA (ssDNA) that is called a presynaptic filament (PF). Through multiple contact points, $\mathrm{PF}$ incorporates the incoming double-stranded DNA (dsDNA) to search for homology and to make a three-stranded DNA alignment, which promotes the switching of pairing and strand displacement (4). The result of this complex succession of events is the initiation of the strand exchange reaction that lies at the basis of homologous recombination in prokaryotes and eukaryotes. At the core of many practical applications of RecA known to date lies this PF-promoted DNA pairing and strand displacement, two main phases of the DNA strand exchange reaction (reviewed in Reference 5).

The most popular in vitro assays of the strand exchange are based on gel electrophoresis of the reaction products (6). However, this approach does not allow for high-throughput analysis and real-time monitoring of the reaction. The latter appears to be possible in DNA probing using fluorescence resonance energy transfer (FRET), one of sensitive reporter methods (reviewed in Reference 7). For example, fluorescein and rhodamine have been used for oligonucleotides labeling in different configurations. These donor-acceptor pairs were successfully applied to assess separately the effects of heterology on the pairing and strand displacement phases in RecA-mediated strand exchange reaction $(8,9)$. Real-time monitoring of the reaction has been carried out due to a special configuration of labeling of single-stranded oligonucleotides (targets) and doublestranded oligonucleotides (probes) by these fluorophores. However, the potential of sensitivity through FRET has not been fully realized in these and other studies $(10,11)$ because the ratio between homologous and heterologous signals was about 2 -fold or lower.

Here, we present an improved RecA-assisted FRET-like assay that enables a high signal-tobackground ratio, about $10-20$ times. Two modifications of the described FRET procedures (8-11) seem to be essential. First, for molecular beacons (12) and displacing probes (13), a nonfluorescent dark quencher 4-(4'-dimethylamino phenylazo) benzoic acid (DABCYL) was used. In the above mentioned hybridization probes, both a fluorophore and a quencher were kept together in close proximity, causing the fluorescence of the fluorophore to be quenched by proximal quenching proceeding via non-FRET-based mechanisms (reviewed in References 7 and 12). Second was the use of triple repeats of the probe sequence as single-stranded targets that resulted in an increase of the effective concentration of homologous ssDNA.
The 28-mer double-stranded hybridization probe used in the study was as follows: 5'-FAM-AAACTAA TAAGATTTACAACAATTTCTC-3', 3'-DABCYL-TTTGATTATTCTAAA TGTTGTTAAAGAG-5', where FAM is 6-carboxyfluoresceine. The 84-mer single-stranded target contained three repeats of the 5'-FAM strand: 5'-AAA CTAATAAGATTTACAACAATTTCT CAAACTAATAAGATTTACAACAAT TTCTCAAACTAATAAGATTTACAA CAATTTCTC-3'. The 101-mer singlestranded oligonucleotides used in the heterologous control were as follows: 5'-TGCAGCGTACGAAGCTTCAGC ACCTAATTGACACCGTACTACTTT AATTAGACCCAGAGACGGAGAG AGAGAGGGAGAGGGAGAGGGAG AGGGAGAGGGAG-3'.

The Escherichia coli RecA protein was purified using the procedure described (14). The $125 \mu \mathrm{L}$ reaction mixture contained $20 \mathrm{mM}$ Tris- $\mathrm{HCl}$, pH 7.5, $2 \mathrm{mM} \mathrm{MgCl}_{2}, 20 \mathrm{mM} \mathrm{NaCl}, 1$ mM ATP, and its regenerating system (3 $\mathrm{mM}$ phosphoenolpyruvate and 30 $\mathrm{U} / \mathrm{mL}$ pyruvate kinase), $10 \mu \mathrm{M}$ ssDNA (in nucleotides), and $3 \mu \mathrm{M}$ RecA. After a 3-min incubation at $37^{\circ} \mathrm{C}$ for $\mathrm{PF}$ formation, the strand exchange was initiated by adding $125 \mu \mathrm{L}$ containing

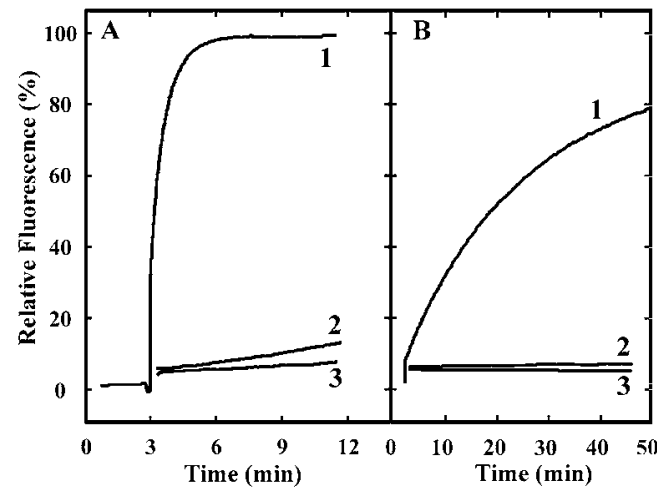

Figure 1. The real-time monitoring of DNA strand displacement promoted by (A) Escherichia coli RecA and (B) Pichia angusta Rad51 proteins. Curve 1 is a full reaction mixture (experiment); curve 2 is the mixture without single-stranded targets (control); curve 3 is the mixture with 101-mer heterologous single-stranded targets (a heterologous control). The reaction was carried out and monitored with a Hitachi F-4000 spectrofluorometer (Hitachi, Tokyo, Japan) at excitation and emission wavelengths of 490 and $520 \mathrm{~nm}$, respectively. Emission spectra were recorded in correction mode by subtracting the spectrum of buffer used. Excitation and emission band passes were set at 10 and $5 \mathrm{~nm}$, respectively. The printed pictures were scanned and processed using Adobe ${ }^{\circledR}$ Photoshop ${ }^{\circledR}$ 8.0. 
$\mathrm{MgCl}_{2}$ (to a final concentration of 12 $\mathrm{mM})$ and a dsDNA probe $(3 \mu \mathrm{M}$, in nucleotides). It is noteworthy that the ratio of ssDNA to dsDNA molecules in the mixture was approximately $1: 1$, whereas the effective concentration of ssDNA (in nucleotides) was about three times more relative to dsDNA. The experiment and two controls are presented in Figure 1A. As expected, both controls showed very low backgrounds. As has been demonstrated earlier (8), the rate of pairing between the single-stranded target PF and double-stranded probes is an order of magnitude greater than subsequent strand displacement. This finding clarifies that the curve 1 in Figure 1A describes, in essence, the kinetics of strand displacement. As shown, approximately a 20 -fold increase of fluorescence (relative to the heterologous control) was observed (at 2-min incubation).

In search for an optimal length of PF, the single-stranded targets of 170, 240, and 343 nucleotides (nt), but containing only one copy of the probe sequence (28 nt) within each oligonucleotide, were used. However, in all three cases the signal-to-background ratio appeared to be about 3-fold lower than that observed for the 84-mer target with a triple probe sequence (the data not shown). It means that the triple repeats are responsible for both the optimal length of PF used in the study and the efficiency of the reaction observed.

This modified FRET-like assay is especially fruitful for study on Rad51 proteins with relatively weak recombinase activities. Rad51 from a thermotolerant yeast Pichia angusta was purified as described (14). The reaction conditions were identical to those used for the E. coli RecA protein. As expected, the $P$. angusta Rad51 protein promoted strand displacement much more slowly than RecA but, as in the previous case, the reaction proceeded with a low background determined by the heterologous control (Figure 1B).

All data presented in Figure 1 were reproducible. The curves presented in the figure are from one typical experiment. The uncertainty in measurements did not exceed $8 \%$.

In conclusion, two modifications of the FRET assay described here including the proximal quenching of nonfluorescent acceptor and a triple repeat of probe sequence in the target allow to increase significantly the sensitivity of the assay. The latter becomes suitable for analysis of separate steps of such a complex mechanism as DNA strand exchange managed by even weak DNA transferases. The described assay was successfully used in analysis of unusual characteristics in such DNA transferases as Rad51 from Chlamydomonas reinhardtii (V. Shalguev, O. Kaboev, I. Sizova, P. Hagemann, and V. Lanzov, unpublished results) and RecAX53, a hyperrecombinogenic E. coli and Pseudomonas aeruginosa chimeric protein (D. Baitin, D. Chervyakova, Yu. Kil, V. Lanzov, and M. Cox, unpublished results).

\section{ACKNOWLEDGMENTS}

We are grateful to $\mathrm{Yu}$. Kil (PNPI) for his help in picture preparation. V.L. thanks Drs. M.M. Cox and D. Baitin for fruitful comments. This project was partially supported by a Fogarty International Research Collaboration Award grant no. 2R03 TWOO 1319-04 to M.M.Cox.

\section{COMPETING INTERESTS STATEMENT}

The authors declare no competing interests.

\section{REFERENCES}

1. Roca, A.I. and M.M. Cox. 1997. RecA protein: structure, function, and role in recombinational DNA repair. Prog. Nucleic Acid Res. Mol. Biol. 56:129-223.

2.Sung, P., L. Krejci, S. Van Komen, and M.G. Sehorn. 2003. Rad51 recombinase and recombination mediators. J. Biol. Chem. 278:42729-42732.

3. West, S.C. 2003. Molecular views of recombination proteins and their control. Nat. Rev. Mol. Cell Biol. 4:435-445.

4. Kowalczykowski, S.C. 2000. Initiation of genetic recombination and recombinationdependent replication. Trends Biochem. Sci. 25:156-165.

5. Volodin, A.A., O.N. Voloshin, and R.D. Camerini-Otero. 2005. Homologous recombination and RecA protein: toward a new generation of tools for genome manipulations. Trends Biotechnol. 23:97-102.
6. Cox, M.M. and I.R. Lehman. 1981. recA protein of Escherichia coli promotes branch migration, a kinetically distinct phase of DNA strand exchange. Proc. Natl. Acad. Sci. USA 78:3433-3437

7.Didenko, V. 2001. DNA probes using fluorescence resonance energy transfer (FRET) designs and applications. BioTechniques 31:1106-1121.

8. Bazemore, L.R., M. Takahashi, and C.M. Radding. 1997. Kinetic analysis of pairing and strand exchange catalyzed by RecA. J. Biol. Chem. 272:14672-14682.

9. Folta-Stogniew, E., S. O'Malley, R. Gupta, K.S. Anderson, and C.M. Radding. 2004 Exchange of DNA base pairs that coincides with recognition of homology promoted by $E$. coli RecA protein. Mol. Cell 15:965-975.

10. Gupta, R.C., L.R. Bazemore, E.I. Golub, and C.M. Radding. 1997. Activities of human recombination protein Rad51. Proc. Natl. Acad. Sci. USA 94:463-468.

11. Gumbs, O.H. and S.L. Shaner. 1998. Three mechanistic steps detected by FRET after presynaptic filament formation in homologous recombination. Biochemistry 37:1169211706 .

12. Tyagi, S., D. Bratu, and F.R. Kramer. 1998. Multicolor molecular beacons for allele discrimination. Nat. Biotechnol. 16:49-53.

13. Li, Q., G. Luan, Q. Guo, and J. Liang. 2002. A new class of homogeneous nucleic acid probes based on specific displacement hybridization. Nucleic Acids Res. 30:e5.

14. Cox, M.M., K. McEntee, and I.R. Lehman. 1981. A simple and rapid procedure for the large scale purification of the RecA protein of Escherichia coli. J. Biol. Chem. 256:46764678 .

15. Shalguev, V.I., Yu.V. Kil, L.V. Yurchenko, E.A. Namsaraev, and V.A. Lanzov. 2004 Rad51 protein from the thermotolerant yeast Pichia angusta as a typical but thermodependent member of the Rad51 family. Eukaryot. Cell 3:1567-1573.

Received 7 March 2006; accepted 10 April 2006.

Address correspondence to Oleg Kaboev, Petersburg Nuclear Physics Institute RAS, 188300, Gatchina, Russia. e-mail: kaboev@omrb.pnpi.spb.ru

To purchase reprints of this article, contact Reprints@BioTechniques.com 